\title{
VIRTUAL SCENE CONSTRUCTION OF LARGE-SCALE CULTURAL HERITAGE : A FRAMEWORK INITIATED FROM THE CASE STUDY OF THE GRAND CANAL OF CHINA
}

\author{
Jian Tan ${ }^{1}$ and Shenghua Wang ${ }^{2}$ \\ ${ }^{1}$ Key Laboratory of Digital Earth Science, Institute of Remote Sensing and \\ Digital Earth, Chinese Academy of Sciences, Beijing, China \\ Tanjian1998@gmail.com \\ ${ }^{2}$ School of Public Administration and Communication, Beijing Information \\ Science Technology University, Beijing, China \\ 386974698@qq. com
}

\begin{abstract}
Virtual reality technology has been applied to the protection of cultural heritage for about 20 years. However, methods or systems of cultural heritage reported in previous studies are still unable to represent large-scale cultural heritage sites such as the Beijing-Hangzhou Grand Canal, the Struve Geodetic Arc and the boundaries of the Roman Empire. We aimed at constructing a large-scale cultural-heritage 3-D model with the focus on better management and organization of the scene. Starting from the case study of the Beijing-Hangzhou Grand Canal, we first explore various remote sensing data suitable for large-scale cultural heritage modeling, and then adopt a 3-D geographic global information system for large-scale 3-D scene organization and management.
\end{abstract}

The entire 3-D virtual scene reconstruction process can be divided into four steps. The first one is the remote-sensing data preparation, where TM, SPOT5 and other remote sensing data were selected according to the characteristics of the cultural heritage of the Grand Canal and further subjected to data filtering and geometric correction. In the second step, the 3-D terrain modeling was carried out based on 3-D earth model segmentation and tile hierarchy system, where we fused and split remote sensing image and sampling spatial information for 3-D terrain model. The third step involves the modeling of local heritage with sophisticated modeling techniques sufficient to build a heritage 3-D model and to integrate terrain model with local scene through aerial orthophotos. Finally, in the fourth step the virtual scene integration is performed in a 3-D spherical system, where we designed a tree nodes system to assembly and manage multi-level and multi-type models of the Grand Canal. After these four steps are completed, the large-scale cultural heritage scene in 3-D spherical information system can be achieved.

Here, we address main challenges in virtual scene reconstruction of large-scale cultural heritage. This study can be valuable for regional and national cultural heritage protection as well as for Chinese government as a reference to infrastructural research, and finally for stimulation of other large-scale cultural heritage research around the world both in 3-D modeling and virtual scene organization.

Jan Zizka et al. (Eds) : CCSIT, SIPP, AISC, CMCA, SEAS, CSITEC, DaKM, PDCTA, NeCoM - 2016 pp. 267-286, 2016. (C) CS \& IT-CSCP 2016

DOI : $10.5121 /$ csit.2016.60123 


\section{KEYWORDS}

Virtual scene construction, large-scale cultural heritage, the Grand Canal

\section{INTRODUCTION}

Virtual reality technology has been applied in the protection of cultural heritage for about 20 years. Zahorik and Jenison [1] suggested that the couple of perception and action is crucial for determining the extent of presence, and mentioned a virtual scene may give the "presence" in ancient cultural heritage. Gaitatzes et al. and Miyazaki et al.[2, 3] presented an overview in modeling cultural heritage through observation. Their efforts were focused on three aspects: how to create geometric models of cultural heritage; how to create photometric models of cultural heritage; and how to integrate such virtual heritage components with real scenes. White et al. [4] proposed an architecture for integrating both the software and the hardware for digitization, management and presentation of virtual exhibitions, whereas Papagiannakis et al. [5] presented a case study of a real-time interactive digital narrative and real-time visualization of an ancient temple. In addition, Christou et al. [6] described the development and evaluation of a large-scale multimodal virtual reality simulation suitable for the visualization of cultural heritage sites and architectural planning. They referred "large-scale" to a haptic interface which was coupled with a realistic physics engine allowing users to experience and fully appreciate the effort involved in the construction of architectural components and their changes through the ages. According to Cabral et al.[7] X3-D is convenient in historic architectural reconstruction so that users might immerse themselves. Bruno et al. [8] summarized the complete methodology by a low-cost multimedia stereoscopic system for digital archaeological exhibition from digitization, management to user interfaces. Núñez Andrés [9] reviewed different techniques including massive capture techniques and traditional survey. They showed the advantages and disadvantages of each technique by applying them to the survey of the great Gate of Antioch.

However, the virtual scene constructions of cultural heritage sites in these studies are still restricted in local area or individual artifact. These methods or systems may create some intuitive 3-D model, but are not yet applicable to the processing of large-scale cultural heritage.

The primary characteristic of large-scale cultural heritage is its huge spatial span, such as Struve Geodetic Arc, the boundaries of the Roman Empire (including the Hadrian's Wall in UK, Der Obergermannisch-raetische Limes in Germany, etc. [10]), the Great Wall or Beijing-Hangzhou Grand Canal of China and so on. Spatial ranges of these large-scale cultural sites extend from hundreds to thousands kilometers.

A large spatial scale of cultural heritages is associated with difficulties in virtual scene construction. Although 3-D scanning and texture photography has been widely used on small objects modeling for many years, it might not be possible to acquire data of heritage spots over area of thousands of square kilometers especially with limited time and funding. Suppose the data are ready, just indexing and processing them could be a tremendous effort. Furthermore, management and rendering of the model output data would likely exceed the capability of ordinary computers. One possibility would be to adopt a Level Of Detail (LOD) concept to reduce data volume, but there are many 3-D model types such as tree, terrain, temples and the like which would hardly keep space consistency and could exceed a roam-able virtual scene. 
How to construct a cultural heritage 3-D model for the large scale and how to manage and organize the scene have been so far difficult challenges not addressed in the literature.

We aimed at solving these two problems by taking the Grand Canal of China as a research subject, using remote sensing data sources for multi-scale modeling of cultural heritage, and adopting a 3-D geographic globe information system as a virtual scene organization platform. This paper explores and presents the overall framework and key steps in virtual scene construction of large-scale cultural heritage.

We first make a brief overview of the Grand Canal of China which is a typical example for a large-scale cultural heritage, and propose the overall roadmap of the virtual environment construction with the four crucial steps: (1) cultural heritage remote sensing data preparation; (2) terrain modeling; (3) local heritage modeling; and (4) virtual heritage scene integration in 3-D global system.

\section{OVERVIEW OF THE BEIJING-HANGZHOU GRAND CANAL OF CHINA}

The Beijing-Hangzhou Grand Canal is the world's oldest and longest canal, and also the largest in engineering scale. It is one of the "Two Great Works" of the ancient China (the other is the Great Wall). Beijing-Hangzhou Grand Canal belongs to a basin-wide cultural site. It connects five drainage basins including the Haihe River, Yellow River, Huaihe River, Yangtze River, and Qiantang River, with a total length of $1794 \mathrm{~km}$, which is 16 times longer than the Suez Canal, and 33 times longer than the Panama Canal. Longer than the namely "the king of canal" the Main Turkmen Canal stretches over $400 \mathrm{~km}$. In chronology, the Grand Canal is the earliest ancient canal which was dug up more than 2,500 years ago, and operated much earlier than the Panama Canal and the Suez Canal. Moreover, the Grand Canal runs through a huge number of ancient cities and other cultural heritages [11] with building age of these heritages from $770 \mathrm{BC}$ to 1900 , including 33 ancient cities and related pier, temples, pagodas, bridges, streets, factories, old kilns and ancient downtowns in 8 Chinese provinces.
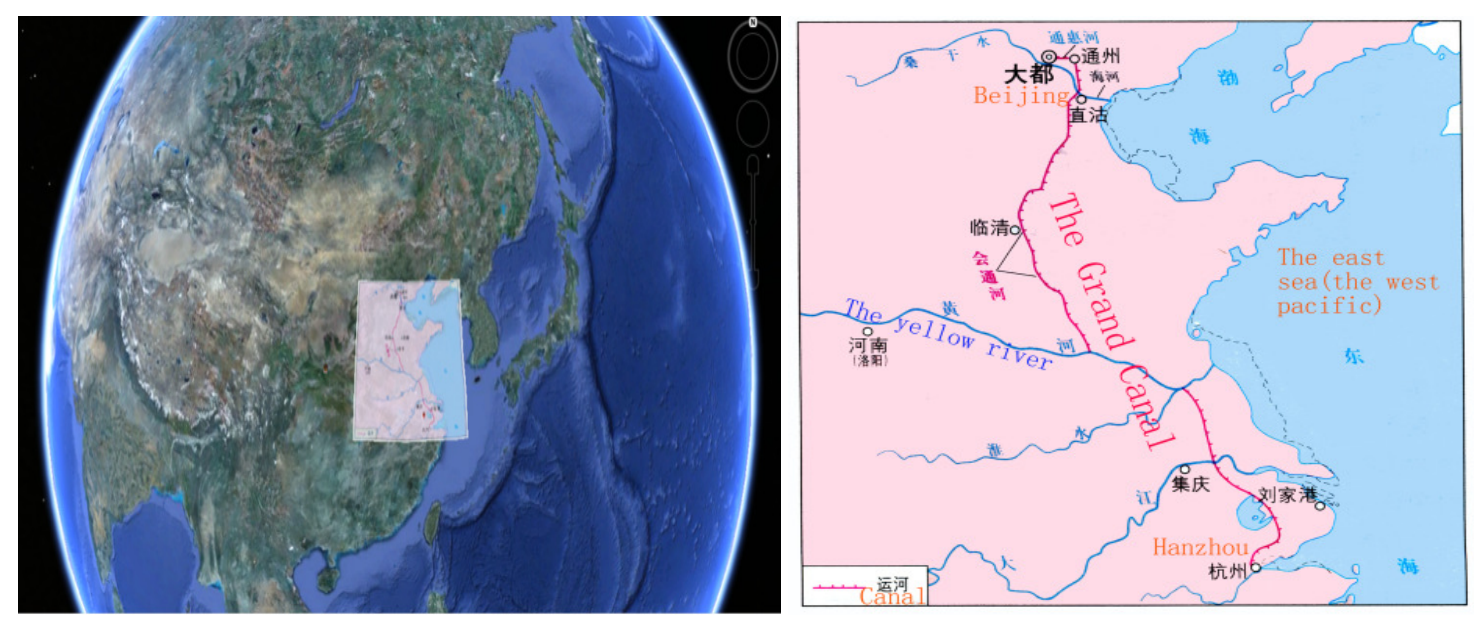

Figure 1. The vast area of the Beijing-Hangzhou Grand Canal

Beijing-Hangzhou Grand Canal is located in China's eastern flat and inhabitable area and its basin area accounts for 10\% of China's land area, while Gross Domestic Product GDP accounts for $25 \%$ of China. The economic development, demand for industrial and residential land is 
rapidly growing, but the environmental pollution is spreading as well. The protection of ancient ruins along the Grand Canal is facing increasing pressure, so the use of virtual reality technology to build virtual scene of the Beijing-Hangzhou Grand Canal is most likely the best means to help securing the Grand Canal Basin heritages, their history and culture.

On the other hand, the virtual scene construction of Beijing-Hangzhou Grand Canal is facing some unprecedented challenges. One of them is the large extent of the Beijing-Hangzhou Grand Canal, which makes it difficult to obtain 3-D data of thousands of kilometers. Another challenge is that the archaeological excavation on the Beijing-Hangzhou Grand Canal will constantly add to the new heritage modeling works, so it would be desirable (although not easy) to provide an open information platform and integrate these and subsequent models into the existing virtual scene.

\section{Methodology}

In order to solve the challenges in the virtual scene construction, we use remote sensing data sources for multi-scale modeling of cultural heritage, and adopt 3-D geographic globe information system as a virtual scene organization platform.

Remote sensing has been aiding the general approach of landscape archaeology since 1970s [12]. However, few studies introduced remote sensing data for 3-D cultural heritage modeling because of their low-accuracy. Here, we see a high potential of remote sensing in large-scale modeling of cultural heritage, particularly as basic data sources for environmental modeling.

It is widely known that GIS could provide an efficient way to integrate archaeological activities, data management, digital object representation and spatial analysis[13]. However, the ability to apply GIS techniques to achieve an integrated management of 3-D data and activities at a large scale is very limited in the field. Therefore, in the case of the Grand Canal and its subsequent 3-D modeling and system integration, we adopt a 3-D geo-spatial global information system, which is closer to digital earth than 3-D GIS, to establish an integrated environment for large-scale cultural heritage.

This virtual environment construction of large-scale cultural heritage based on remote sensing data sources and 3-D global geo-spatial information system can be divided into four parts in time sequence. These steps are: (1) remote sensing data preparation; (2) terrain modeling; (3) local cultural scene modeling; and (4) virtual heritage integration in 3-D global GIS. In the next sections, the contents and main issues of each step are analyzed in detail.

The overall research flow-diagram is shown in Figure 2 below: 


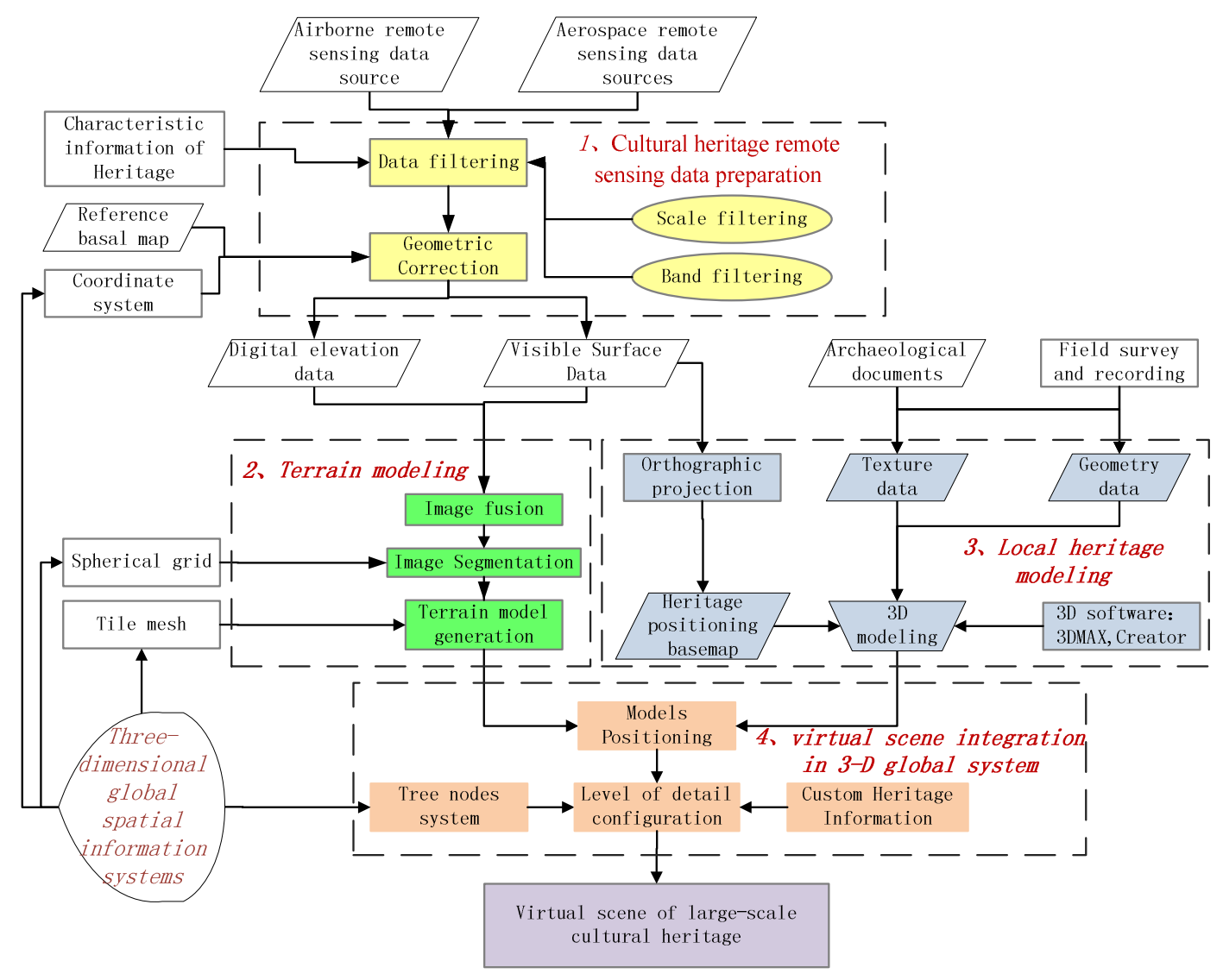

\subsection{Remote sensing data preparation for cultural heritage}

Heritage remote sensing data preparation work is divided into two parts, data filtering (or data) and geometric precision correction.

Currently remote sensing has formed a multi-level, multi-angle, multi-field observation system from the ground to the air, and even space. From the 1960s onwards, technologies such as thermal infrared imaging, airborne synthetic aperture radar, multi-polar surface-penetrating radar and high-resolution space borne synthetic aperture radar have become sophisticated. Remotesensing spectral bands from the earliest visible expand to near-infrared, shortwave infrared, thermal infrared, microwave direction, and these spectral extensions will adapt to a variety of data acquisition for material composition and geometrical shape. Synergies of large, medium and small satellites and combinations of high, medium, low orbits provide data which form a complementary series for specific domain with temporal resolutions ranging from a few hours to 18 days.

The remote sensing technology can nowadays provide more spatial information than ever before, but not all of the remote sensing data sources carry the characteristic data of spatial objects in desired location. Therefore, in the large-scale modeling study of cultural heritage, a proper selection is needed of the remote sensing data to express object characteristics of cultural heritage. Besides the simple judgment by spatial region and time stamp, the selecting work of remote sensing data can be divided into scale filtering and band filtering. 
Scale is the key for understanding the complexity of spatial objects and is regarded as one of the standards of spatial information representation. Katsianis et al. [14] defined four kinds of scales associated with spatial phenomena, one of which is spatial resolution. An appropriate spatial resolution can reflect characteristics of the spatial structure of a specific target. Spatial resolution of remote sensing image represents the level of spatial detail and ability to separate spatial subject and its background, and also reflects the information hierarchy of the earth surface. For example, the riverway of the Grand Canal is $80 \mathrm{~m}$ wide on average, so $30 \mathrm{~m}$ resolution remote sensing data can be adequate for the riverway research and information extraction, but the same 30 meters remote sensing images are unable to express ancient buildings which are 10 meters wide on average. Therefore, in our study we selected the remote sensing image of optimal spatial resolution for expressing particular spatial objects inside the Grand Canal region according to their own characteristics(Table 1).

The Grand Canal cultural site is a complex spatial object with multi-scale structure, inside which objects have different spatial ranges. Thus, depending on the characteristic scale of the internal objects, we filtered remote sensing data sources at the first approach at virtual environment construction for the Grand Canal.

Table 1. The introduction of candidate remote sensing data

\begin{tabular}{|l|l|l|l|}
\hline $\begin{array}{l}\text { Data } \\
\text { type }\end{array}$ & introduction & $\begin{array}{l}\text { average } \\
\text { spatial } \\
\text { resolution }\end{array}$ & $\begin{array}{l}\text { sensitive spatial } \\
\text { objects in the Grand } \\
\text { Canal cultural site }\end{array}$ \\
\hline TM & $\begin{array}{l}\text { U.S. Land Observing Satellite, three of seven } \\
\text { observation bands are visible bands }\end{array}$ & $30 \mathrm{~m}$ & $\begin{array}{l}\text { riverway of the ancient } \\
\text { canal, lakes along the } \\
\text { basin }\end{array}$ \\
\hline SPOT5 & $\begin{array}{l}\text { French Earth Observation System (Systeme } \\
\text { Probatoired'Observation delaTarre[15],provides } \\
\text { 5 observations bands and panchromatic image }\end{array}$ & $10 \mathrm{~m}$ & $\begin{array}{l}\text { change information of } \\
\text { canal basin landscape } \\
\text { (land types) }\end{array}$ \\
\hline SRTM1 & $\begin{array}{l}\text { Shuttle Radar Topography Mission, provide } \\
\text { terrain data from 60 degrees north latitude to 60 } \\
\text { degrees south latitude with a total covering area } \\
\text { of more than 119 million square kilometers }\end{array}$ & $30 \mathrm{~m}$ & $\begin{array}{l}\text { the terrain topography } \\
\text { along the Grand Canal }\end{array}$ \\
\hline $\begin{array}{l}\text { Airborne } \\
\text { Remote } \\
\text { Sensing }\end{array}$ & $\begin{array}{l}\text { images are taken by airborne remote sensor, } \\
\text { have higher spatial and temporal resolution, but } \\
\text { narrower in spatial range comparing with } \\
\text { satellite images Airborne photos }\end{array}$ & $0.2 \mathrm{~m}$ & $\begin{array}{l}\text { contours of buildings } \\
\text { and structures, roads } \\
\text { and landmarks along } \\
\text { the Grand Canal }\end{array}$ \\
\hline
\end{tabular}

Band filtering is a necessary step in the case of multi-spectral remote sensing data supplies, which requires selecting the best band or band sets to extract spatial objects and express spatial information effectively. Generally a remote sensor is only sensitive to specific spectral wavelength range which is named band. The original remote sensing images are all single band. In practice, according to three-color synthesis principle, the digital image processing systems place three different bands of remote sensing images on three channels which are assigned to red, green, blue to form a color image[16]. Specific details of band assembly are shown in Figure 3 . 


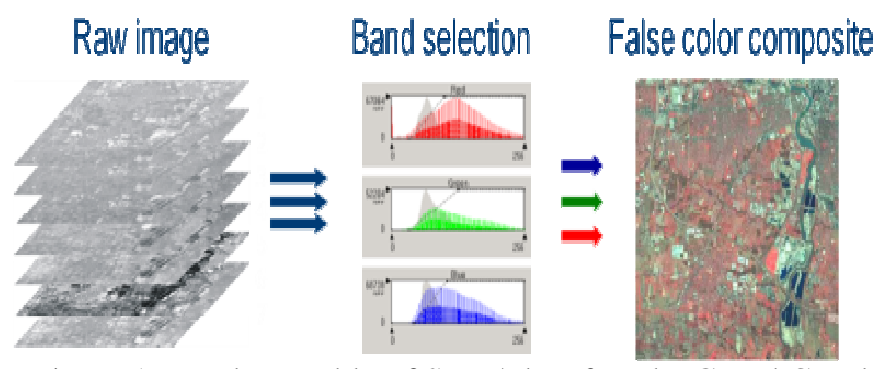

Figure 1. Band assembly of Spot5 data for The Grand Canal

There are three main principles for the best band selection:

(1) The largest amount of information it carries; (2) the smallest possible correlation among bands; (3) the largest difference among spatial object types which the band is sensitive to. The best band combination is three bands with abundant information content, the smallest correlation and distinguishable sensitive spatial objects [17]. However, despite each band's own characteristics, there are more or less information duplication and redundancy among hyperspectral remote sensing data. Consequently, the selection should be based on the spectral characteristics of observation targets. For example, TM1,4,5 would be the best band combination for settlements and waters interpretation[18], while TM3,4,5 would be the best band combination for farmland, woodland, grassland interpretation[19]. When using remote sensing data to construct a 3-D scene of cultural heritage, selection of band combinations should be based on the characteristics of spatial objects inside the heritage site.

The core heritage trail and main observation target of the Grand Canal is the riverway, of which the main body is water. The spectral characteristic of water depends on its material composition, but also reflects the various water states. Natural water bodies have significantly higher absorption for $0.4 \sim 2.5 \mathrm{Lm}$ electromagnetic wave than most other surface features [20]. In the infrared, water absorbs more energy than in visible light, while vegetation, soil in the two bands have smaller energy absorption, and a higher reflection, that results in significant difference between the water and the vegetation or soil in the two bands. Although the panchromatic band is not more sensitive to the Grand Canal water body than other spatial objects, the panchromatic band provides a high spatial resolution of $2.5 \mathrm{~m}$, which has a good effect for the spatial information expression of the overall Grand Canal region. For these reasons, we chose the near infrared (XS3), mid infrared (B4), and panchromatic band (PAN) for remote sensing image synthesis of the Grand Canal heritage(Table 2).

The purpose of the geometric correction is to correct errors caused by non-systemic factors, and is based on mathematical models of geometric calibration. This correction is also a process which combines translation and rotation to project image onto a plane through homonymy points from remote sensing images and underlay reference map to place the spatial objects right on the corresponding reference position after correction [21].

In the Grand Canal remote data preparation, we used the method of least squares to calculate imagery geometric correction, in which $(\mathrm{t}+1) \times(\mathrm{t}+2) / 2$ equals to 6 control points similarly to as described elsewhere [22]. In order to promote accuracy, in each image we select at least 15 control points for image registration. Generally there are two principles in control point selection for large-scale cultural heritage:

(1) The control points should be evenly distributed over the entire heritage site. 
(2) The control points should optimally be the corner points of a permanent feature rather than of any removable points, such as docks or road crossings in the case of the Grand Canal.

Table 1. The bands features of SPOT5 sensor

\begin{tabular}{|l|l|l|l|}
\hline $\begin{array}{c}\text { Spectral } \\
\text { band }\end{array}$ & $\begin{array}{c}\text { wavelength } \\
\text { range }(\boldsymbol{\mu m})\end{array}$ & $\begin{array}{c}\text { ground } \\
\text { resolution } \\
(\mathbf{m})\end{array}$ & \multicolumn{1}{c|}{ The main application areas } \\
\hline XS1:Green & $0.50 \sim 0.59$ & 10 & $\begin{array}{l}\text { This band has part transmission in water body and } \\
\text { great reflectance in healthy green plants, it can } \\
\text { distinguish vegetation types and assess crop } \\
\text { growth. }\end{array}$ \\
\hline XS2:Red & $0.61 \sim 0.68$ & 10 & $\begin{array}{l}\text { This band provides measurable plants chlorophyll } \\
\text { absorption rate, and in turn plant classification, can } \\
\text { distinguish artificial feature types too. }\end{array}$ \\
\hline $\begin{array}{l}\text { XS3: near } \\
\text { infrared }\end{array}$ & $0.78 \sim 0.89$ & 10 & $\begin{array}{l}\text { This band is greatly absorbed in water and is used } \\
\text { to draw water body boundaries, detect the content } \\
\text { of aquatic organisms. }\end{array}$ \\
\hline $\begin{array}{l}\text { B4: mid } \\
\text { infrared }\end{array}$ & $1.58 \sim 1.75$ & 20 & $\begin{array}{l}\text { Usually vegetation, water, soil have obvious gray } \\
\text { level difference in this band, so this band is used to } \\
\text { detect vegetation, water content and soil moisture, } \\
\text { and distinguish the difference between clouds and } \\
\text { snow. }\end{array}$ \\
\hline PAN & $0.48 \sim 0.71$ & 2.5 & $\begin{array}{l}\text { With the highest spatial resolution in SPOT5, this } \\
\text { band can be used for forestry research and } \\
\text { planning, urban planning and large scale thematic } \\
\text { mapping. }\end{array}$ \\
\hline
\end{tabular}

\subsection{Terrain modeling of heritage site}

Heritage site terrain modeling refers to processing of remote sensing data of large-scale cultural heritage into a 3-D terrain model so that information systems can load and render as backgroundmodel of the virtual scene. It is an important step of the large-scale cultural heritage modeling. On the one hand, numerous reports $[23,24]$ suggested that the terrain features are closely related with formation and development of large-scale cultural heritage areas. On the other, with 3-D terrain models, viewers could analyze and rebuild the ancient ruins more intuitively, and present more reasonable explanation of different cultural phenomena from archaeological surveys and excavations than without them [25].

The terrain modeling study is based on 3-D global information systems. The main reason for the system adoption is no limitation on the scale of possible terrain model which may cover a huge spatial range.

Terrain modeling includes image fusion, image segmentation and terrain model generation three steps, and how to perform these steps is determined by 3-D Geographic Information System 3-D Earth model structure. Therefore, before terrain modeling work, an introduction to 3-D Earth model is necessary (Figure 4 ). 


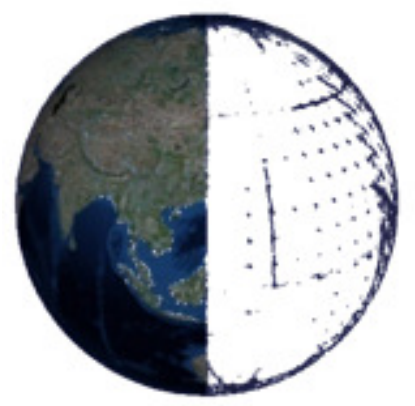

3-D Earth model

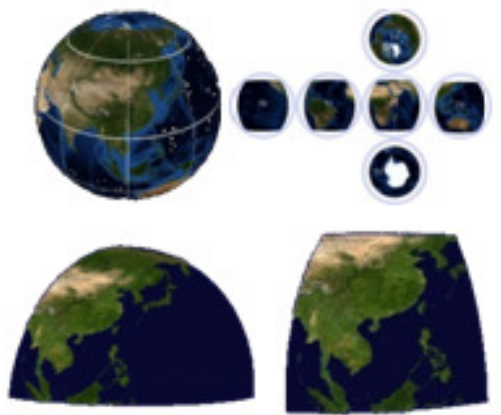

Spherical grid and Tile-hierarchy in 3-D Earth model

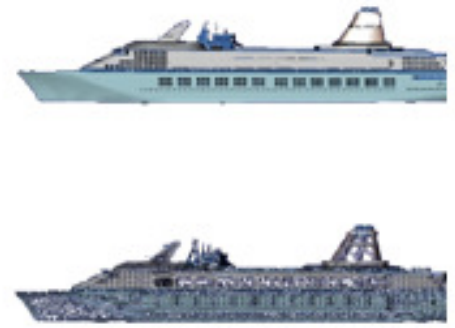

ordinary 3D model

Figure 2. The comparison between 3-D Earth model and ordinary 3D model

3-D Earth model, which is a combination of multiple parts, differs from an ordinary 3-D model. The division rules can be classified into two categories: spherical grid and tile hierarchy. Purpose of the both is to reduce the model data throughput during system operation, but they are not the same in principles and functions.

Spherical grid is a seamless mesh system of the Earth ellipsoid surface with the classic subdivisions called graticules. Cells in spherical grid could be uniform or non-uniform (irregular). Clearly, the morphology of uniform spherical grid cell is more stable with regular borders, and is more convenient to prepare and assembly 3-D data in the 3-D Earth model than that with the irregular borders.

Tile-hierarchy is a hierarchically organized system of spatial data with each tile in the hierarchy being a specific 3-D model. Basic idea of tile-hierarchy is to subdivide a parent tile into a number of child tiles under same data volume constraint, the smaller range a tile covers, and at the higher resolution it could possess [26]. Owing to this subdivision, when spatial data are required, an appropriate tile with the closest scale is delivered so that to reduce the access time and data load during User-System interaction.

As a concrete 3-D model, 3-D Earth tile is not only the direct target for spatial data integration, but also the environmental information carrier in the case of the Grand Canal. We were able to seamlessly integrate spatial data or 3-D models into the tile.

Tile in 3-D Earth model, similarly to other 3-D visualization models, is composed of two parts: geometry mesh and texture. The mesh information and texture information in tile are derived from existing spatial data. Tile mesh is used to represent geometrical shape of the earth surface with each vertices in the mesh expressed in spherical coordinates $(\mathrm{r}, \theta, \varphi)$. Geometric resolution of tile means separation of the zenith angle $(\theta)$, azimuth angle $(\varphi)$ between tile vertices. The radial distance (r) of tiles vertex in earth science field usually refers to elevation value which is derived from remote sensing data products such as elevation SRTM in this case. The tile texture is used to indicate shape and color of earth surface, in which case the tile texture is the visible spectrum data from the hyper spectral remote sensing image such as SPOT5 or TM.

Given the basic platform and the structure of the 3-D earth model, the 3-D terrain modeling process is to assign spatial information from remote sensing data sources into tile vertex.

Firstly, image fusion here was used to integrate different spatial data source into one tile vertex with predefined weights. In the case of the Grand Canal modeling, we based on the same UTM 
coordinate system fuse TM remote sensing images; the weight of overlapping portion is the arithmetic mean, as shown in Figure 5:

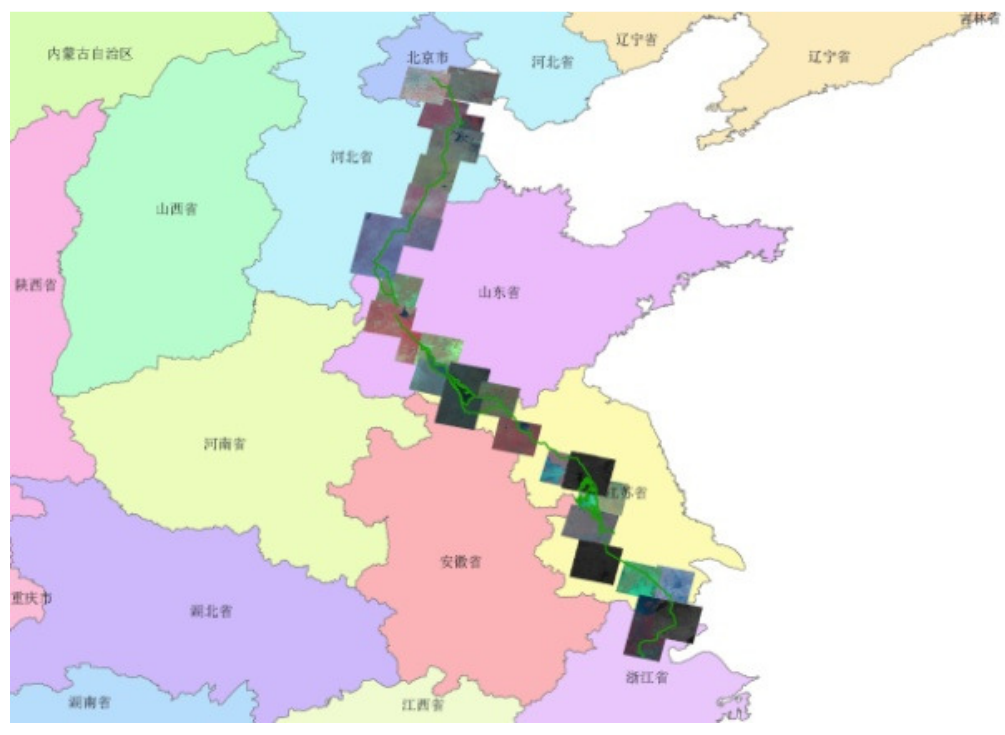

Figure 3. The Spot5 and TM data fusion along the Grand Canal

Secondly, remote sensing image segmentation must be consistent with the borders definition of spherical grid and tile hierarchy, as otherwise it may cause terrain dislocation in the entire virtual scene. In the case of the Grand Canal modeling, in accordance with the tile with 10 meter spatial resolution and $30 \mathrm{~km}$ side-length, we cut TM5 images and elevation images as demonstrated in Figure 6:
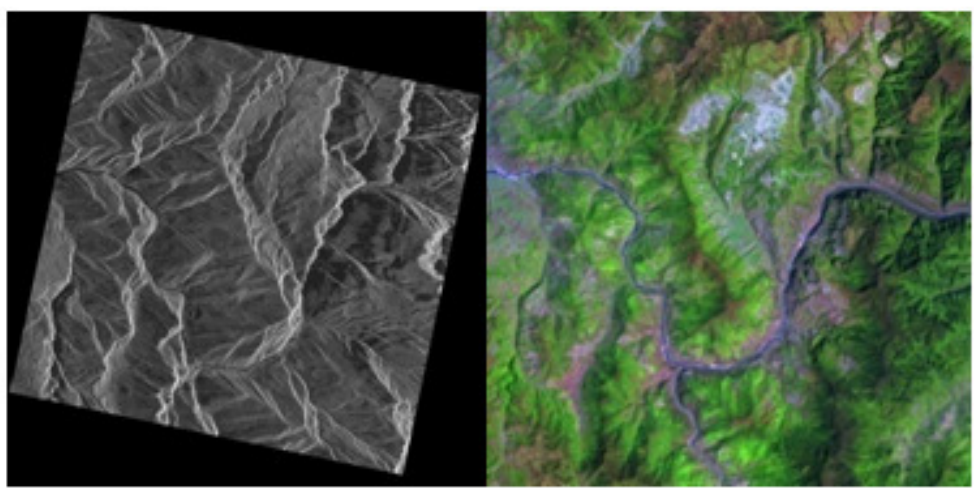

Figure 4. Segmentation of TM5 image and elevation image(rotated) according to the tile boundary

Thirdly, depending on predefined tile meshes of each scale, we sampled remote sensing images to every tile vertex. The generation process of a specific 3-D terrain model of a heritage site was as presented in Figure 7 and linear interpolation of vertices to fill the final model was as shown in Figure 8. 


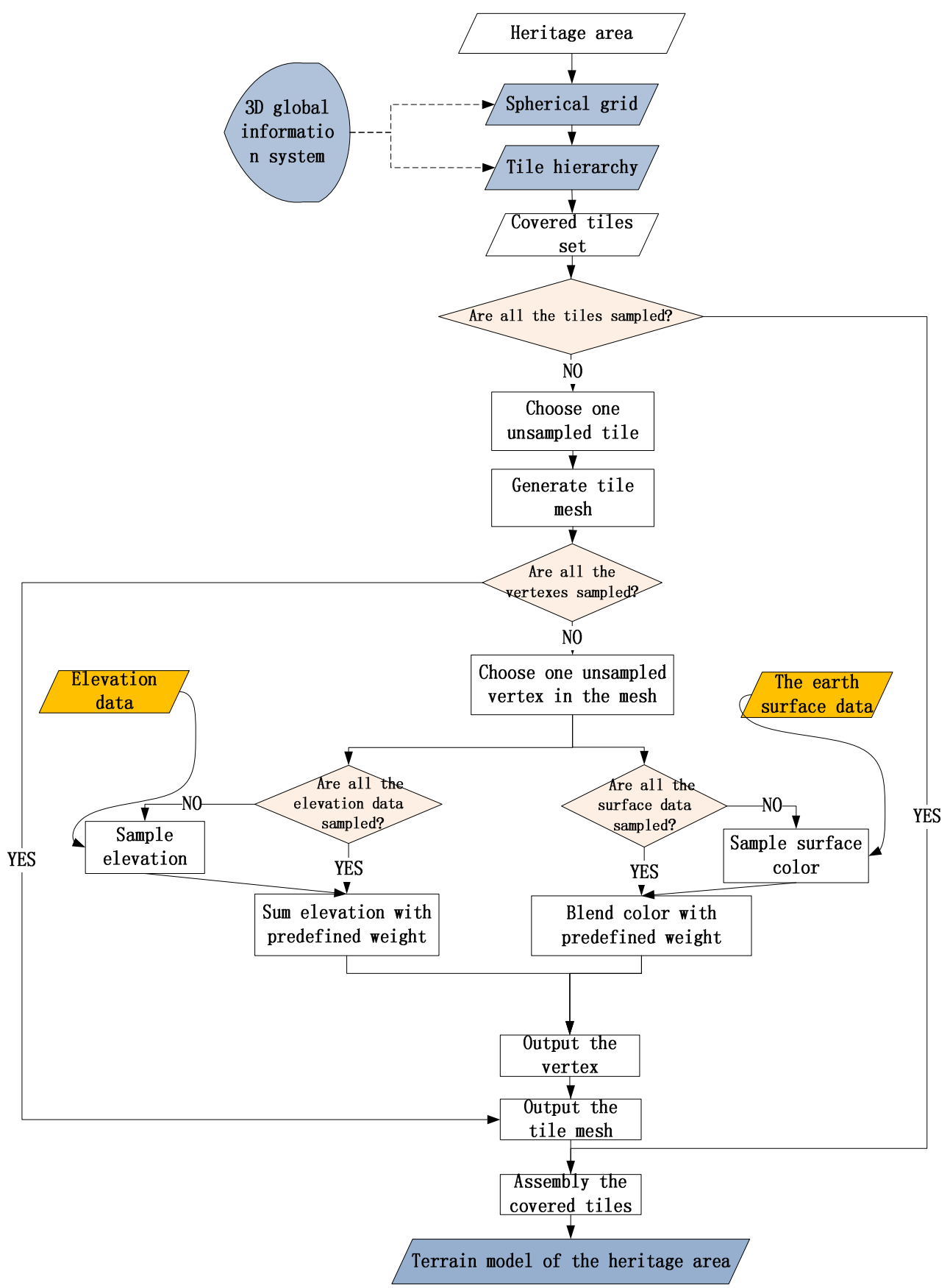

Figure 5. Generation process of a specific 3-D terrain model 


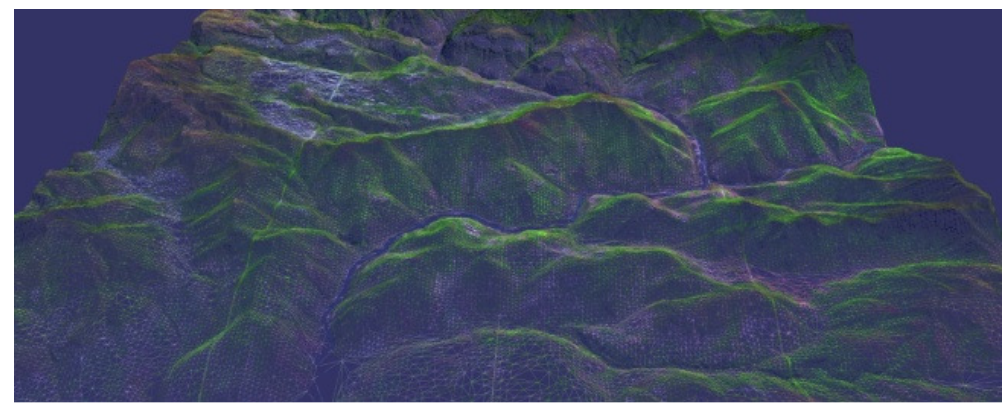

Each vertex of tile mesh has sampled color and elevation from remote sensing data

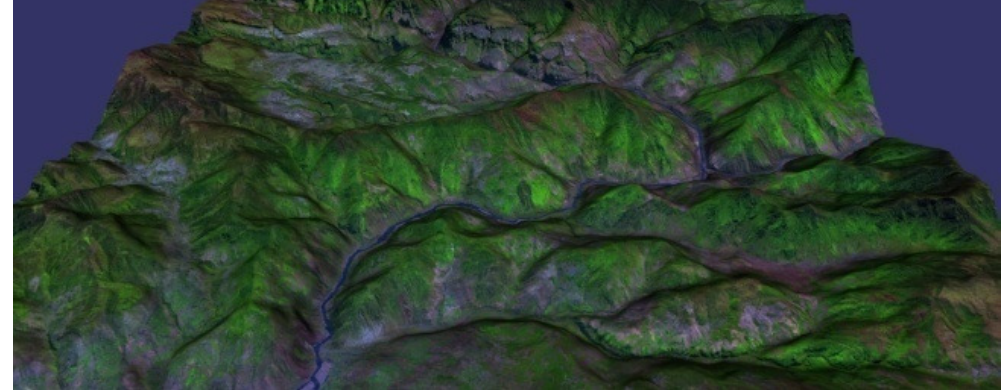

Linear interpolation of vertexes to fill the final model

Figure 6. 3-D terrain modeling based on previous image segmentation

\subsection{Local heritage modeling}

Local heritage refers to centralized heritage places in the large-scale cultural heritage area. Compared with terrain modeling, local heritage modeling has two differences:

One difference is the higher spatial resolution of a model. A 3-D terrain model is under the resolution limitations of remote sensing data that the maximum is not more than 0.2 meters, while in local heritage modeling where we can use a laser radar or a high-precision camera for data acquisition, the spatial resolution can reach even centimeter or millimeter resolution level;

The other difference is a richer type of modeling objects in local heritage. Although a 3-D terrain model covers a wide range, its main purpose is mostly to provide a natural background model which brings basic information on topography and landforms. While the local heritage modeling includes a variety of static spatial entities in cultural heritage area, specific modeling objects includes buildings, vegetation, bridges, piers, heritage and other artificial or natural objects.

Local heritage modeling uses mainly a 3-D model of small-scale cultural heritage. So far its data collection and modeling process, data integration and key technical problems have been thoroughly discussed in many previous studies. For example, Akca [27] used a structured light system for data acquisition, Carmel et al.[28] classified entities of cultural heritage for targeted digitalization, and Alsadik et al.[29] presented a camera network for image-based modeling of cultural heritage. Hug and Gonzalez-Perez [30] qualitatively evaluated three modeling techniques derived from information system engineering to represent cultural heritage domain concepts. More detailed technology of local heritage modeling has been described elsewhere $[2,6,23,31$, 32]. 
Both local heritage models and 3-D terrain models are parts of the large-scale virtual scene. After local heritage modeling, different models in geographic global information system need to be integrated. This integration is solved by using the orthographic projection (Figure 9).

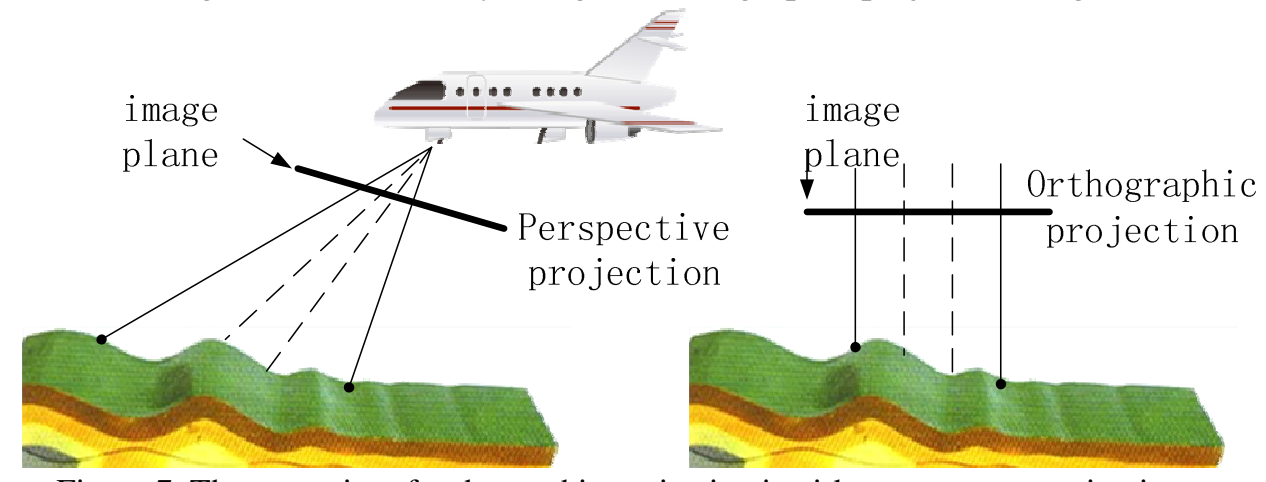

Figure 7. The necessity of orthographic projection in airborne remote sensing image

The projection of an airborne remote sensing image is the central projection which is associated with distortion. Because photosensitive surface tilts and undulating ground generate point displacements in an image, raw airborne images cannot objectively and accurately indicate the shape and location of spatial features. The images have to be processed in order to obtain orthophotos, which are the photographs orthographically projected, and the process is called ortho-correction [33].

Orthophotos, in particular airborne orthophotos are important basal data of cultural heritage 3-D modeling. They have high spatial resolution, allowing for clear identification of buildings, and silhouette of landmarks. As airborne orthophotos are perpendicular to the ground, they reflect the true ground position and topological relations, which can provide spatial orientation and positioning information for high-precision 3-D model to guarantee the accuracy of 3-D spatial measurement and analysis. The import to the 3-D global information system is presented in Figure 10.

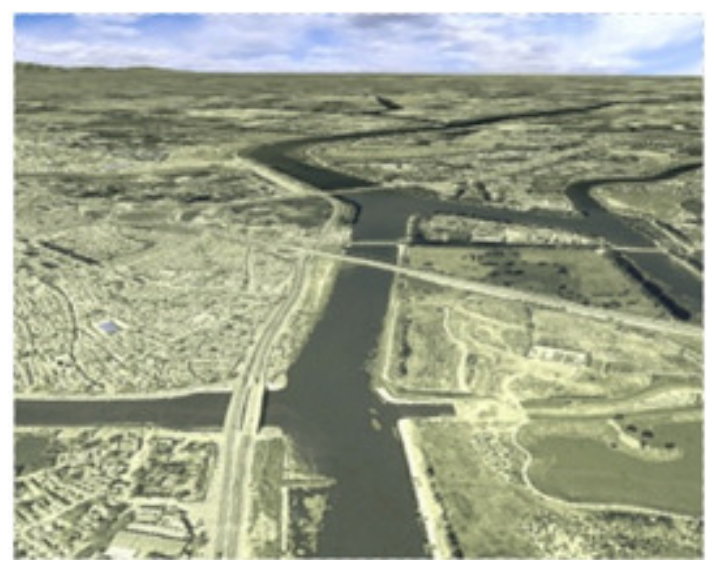

Figure 8. Airborne remote sensing image import into 3-D global information system

As shown in Figure 11, we obtained an airborne remote sensing image of the ancient canal sluice ruins after ortho correction. This led to elimination of the shadows and each pixel on the image regaining its right place within geographic coordinates. Thus, not only the corrected image can be imported into a 3-D modeling software such as Basal Map, 3-Dmax or Creator, but it can also be 
used as a texture layer, for the terrain model to sample from. After integration in 3-D global information systems, the identical geographic coordinates provided by orthophoto become the key bridge between 3-D earth model (terrain model) and the heritage virtual scene, so the high precision of orthophoto ensures accuracy and consistency of the integration.
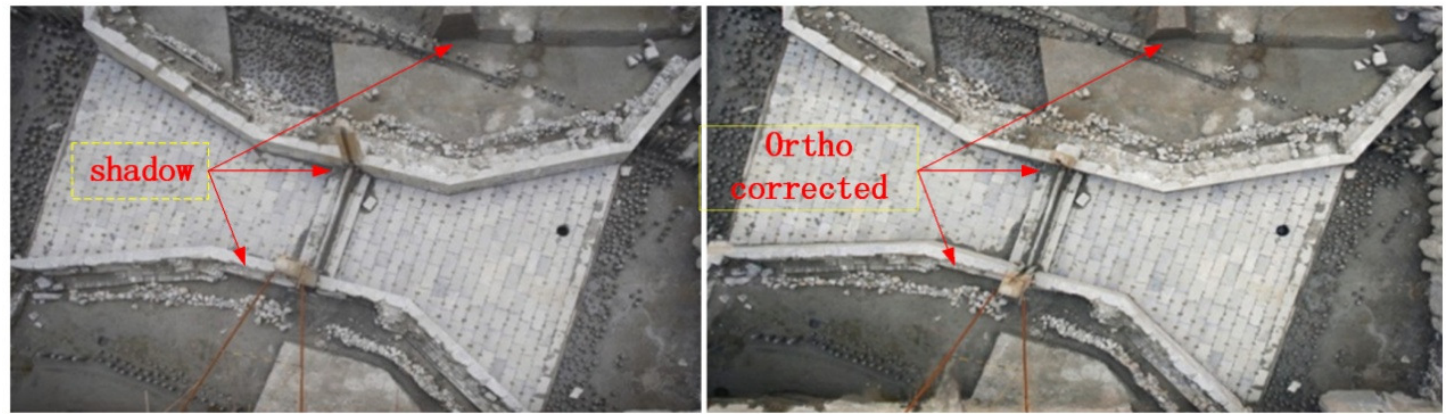

Figure 9. Airborne remote sensing image of a sluice on the Grand Canal before and after orthographic projection

Orthophoto production often requires special equipment. In the case of central projection aerial photographs of flat ground using a mechanical optical instrument could be sufficient. On an undulating ground or a bumpy flight, orthographic projection device such as an opticalmechanical differential rectification instrument and computer numerically controlled analytic projector is available. In the case of dynamic remote sensing which have not captured predefined parameters, all-digital correction machine is usually used to produce the orthophoto[34].

As shown in Figure 12, we located the specific local heritage model based on airborne orthophoto in the 3-DMax software.

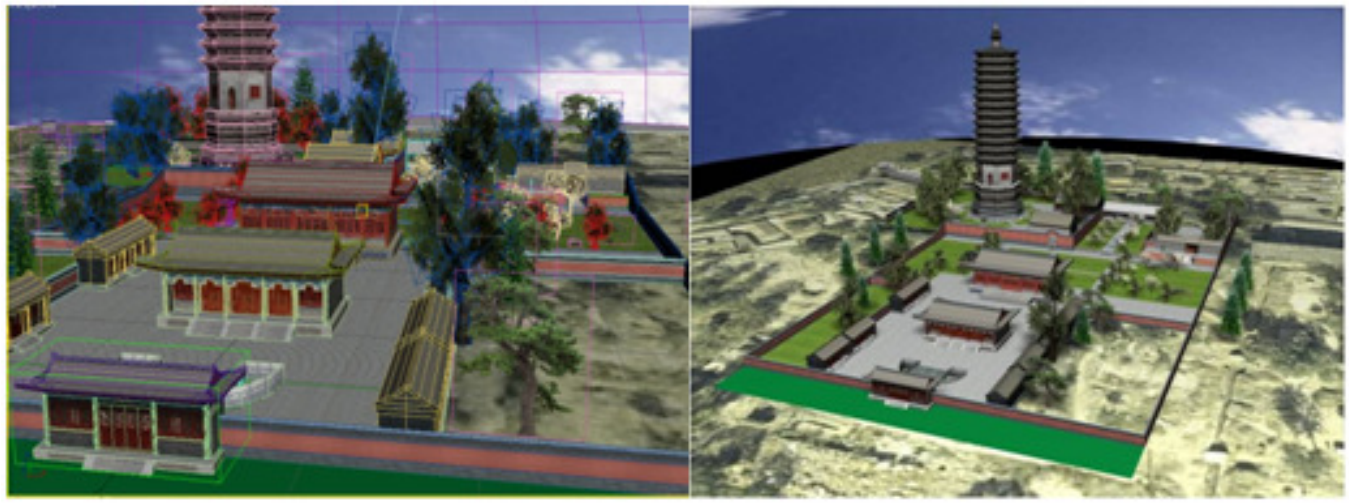

Figure 10. Local heritage modeling and positioning based on Orthophoto in 3-D Max

\subsection{Virtual scene integration in 3-D spherical system}

In the final integration step, in order to embed heritage models into 3-D global information systems seamlessly, we have established an integrated tree of 3-D models (Figure 13). 


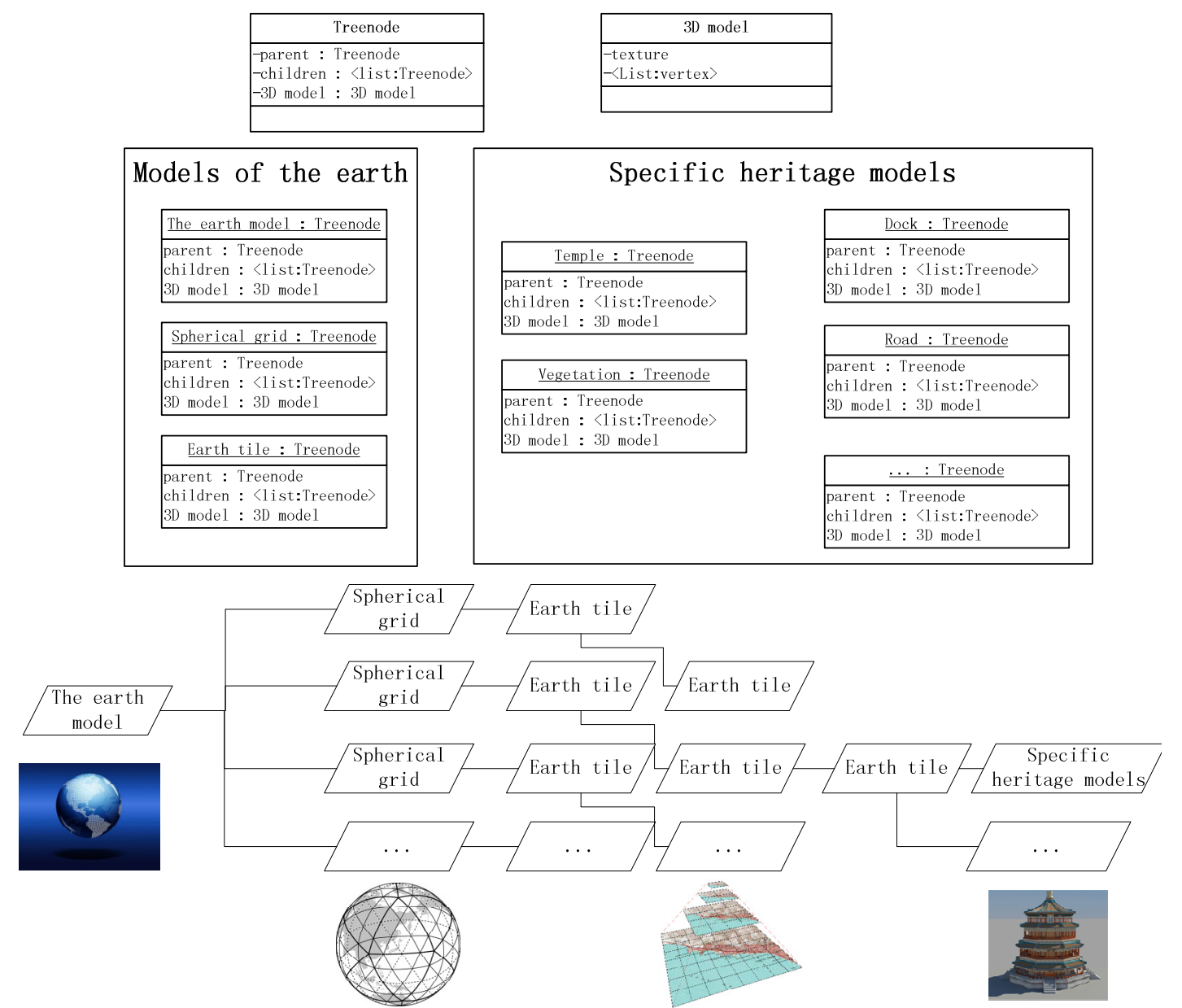

Figure 11. The integrated tree system for 3-D virtual heritage scene organization in 3-D global information system

A tree node object encapsulates each 3-D model, and it may have no more than 1 parent node and unlimited child nodes. This treelike organization for large-scale virtual scene has three characteristics:

(1) All levels of spatial objects are under identical geographic coordinate system

(2) Spatial ranges of all child nodes do not exceed the parent tree node.

(3) In LOD configuration, models-switch from the parent node to child node by viewpoint movement is seamless. It means there is no place to see both the parent and its child nodes and there is also no place to see nothing at heritage site.

This treelike organization has two advantages for large-scale heritage scene:

(1) The data object and user interface definitions in heritage scene are uniform. Every 3-D model of mutli-scale or different types are all tree nodes, such as terrain, roads, buildings, lights, controllers, triggers, particle systems, etc. It helps achieving flexibility in virtual scene assembly so that each node can be assigned to one another as parent or child. Through a consistent interface, operations like node add, delete, property editing and others are very convenient. 
(2) Tree level reflects spatial cover. This implicit spatial description makes it simple and easy to understand spatial relations among 3-D models. Through the view of nodes' bounding box, we can intuitively choose operations including add, delete, edit for virtual scene assembly. Furthermore, each tree node could have its custom properties which are stored in attribute list that promotes the extensibility of the virtual scene to present extra information about history and culture.

\section{RESULTS}

The results from the present study can be divided into two aspects. One was the establishment of a presentation platform for integrated large-scale natural and cultural heritage. The other was to assemble the terrain models of the entire Grand Canal with local heritage models of Jining(ancient city), Tongzhou(ancient city), and the Dragon King Temple which located at the junction between the Grand Canal and Wenshui river. The average distance between these three local heritages was $300 \mathrm{~km}$.

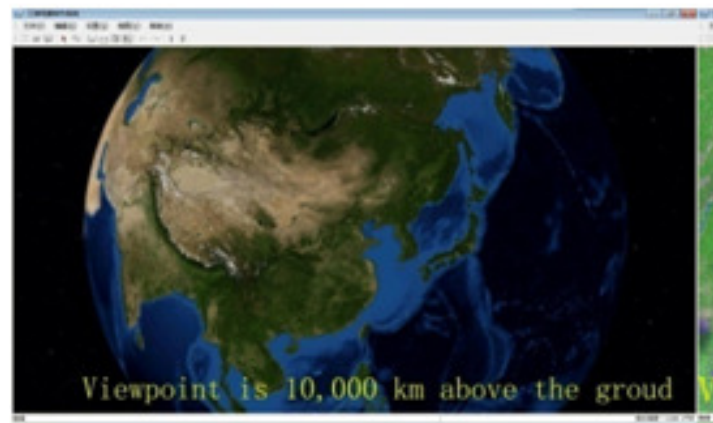

a) The whole blue marble

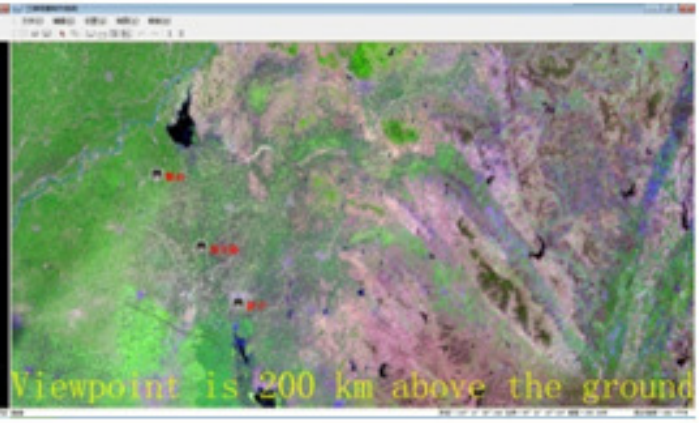

b)The overview of the Grand Canal

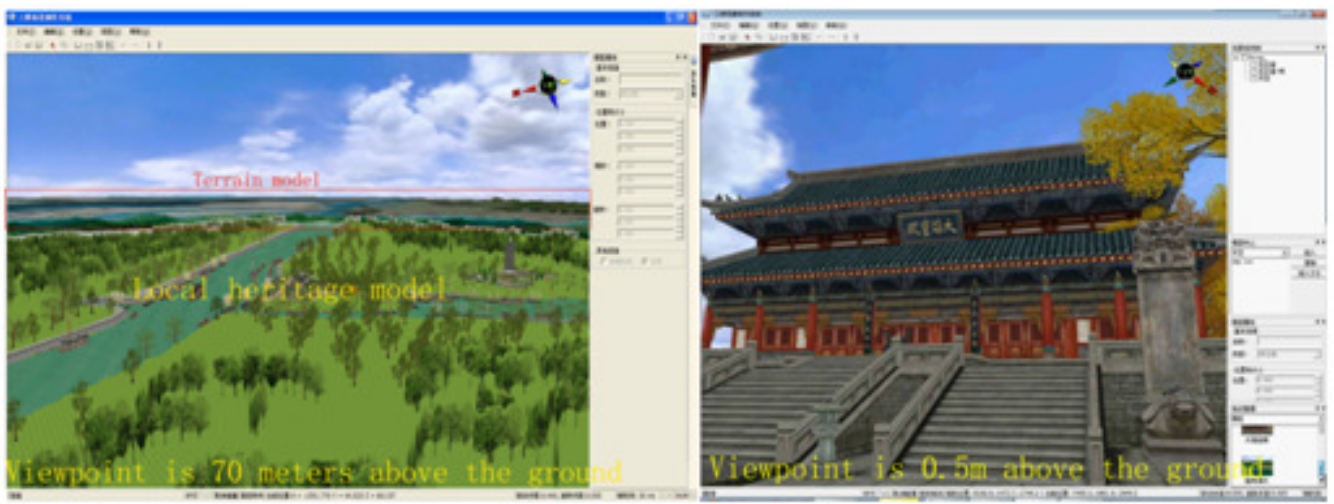

c) Flying to a local heritage(Yangzhou city)

d) A close look to an ancient temple

Figure 14 The integrated scene of the Grand Canal in the 3-D global information system

\section{DISCUSSION}

In order to establish three-dimensional model of large-scale cultural heritage for the present study data selection, processing, combination with a 3D Earth model, integrated management of 3D GIS and a comprehensive workflow were completed. 
One novel thing about the study is that the remote-sensing data source was introduced. In the modeling of cultural heritage this type of data source is not typically used. The reason is that it is collected from too far away, and the accuracy of the data cannot reach the standard for 3D modeling. In addition, the shooting angles almost do not reflect the information on the sides of cultural heritage at all[35]. However, here we found advantages of remote sensing data source in modeling of large-scale cultural heritage.

For example, remote-sensing data source can provide spatial information that reflects various types of surface features in the cultural heritage. The reason is that the existing remote sensors cover a rather wide wavelength range, and for any surface feature, corresponding sensitive data can be found.

Remote sensing data sources can help to achieve quickly 3D modeling of large-scale terrains and landforms. This is particularly important in the modeling of large-scale cultural heritage that crosses hundreds of kilometers.

Therefore, based on the electromagnetic wave reflection characteristics of cultural heritage, after careful data screening and matching, remote sensing data can easily provide the information needed for modeling of cultural heritage.

Another development of the present study was that the 3-D global GIS was used for integration of scenes of cultural heritage at a large scale although this strategy was originally inspired by Google earth experience. In the 3D GIS, the space that can be explored is unlimited. In addition, differences in the spatial scales of terrains and surface details can be directly viewed.

When the present project was launched in 2007, Rome Reborn in Google Earth (RR project)[http://romereborn.frischerconsulting.com/project_news.php] was not yet on line. Although our ultimate interface looks very similar to that of Google Earth, there are certain differences. Firstly, in the RR project[36], established models are released to Google earth as kml layer file, the information on cultural heritage can only be linked through the web, and visualization of the information is prioritized. In our project, for the scenes of cultural heritage a more specialized professional structure was adopted. The cultural heritage model and the 3D Earth model were integrated using a tree structure. In addition, at each node aside from the 3D visual information, custom culture, heritage information can be added. In this way, management and retrieval of cultural heritage information can be further developed. Secondly, the paper [36] published from the RR project did not disclose the method used to prepare spatial data and process of integrating the Earth model and the cultural heritage model. This may be because as two different groups were responsible for modeling the Earth and modeling the ancient buildings, the information available to the author was incomplete.

Although our modeling developments may have still some potential for improvement, we find our study a robust and accurate representation which works extremely well for the spatial features of the Grand Canal. All parts of our final result focus on the Grand Canal heritage, allowing users to better understand the entire picture of the Grand Canal heritage. While the 3D model integrated in the RR project was focused on the scope of the ancient city of Rome, which is a relatively small-scale cultural heritage, in our project, processing of remote sensing data terrain modeling over thousands of kilometers was completed and three local heritage models with 300 $\mathrm{km}$ space between were also established. 
Due to limited funding and staff, currently we have only over 200 high-precision threedimensional models. Nonetheless, our study provides a basic framework, and an import interface for new models. This will provide continuous support for the presentation and protection of the Grand Canal heritage in the future.

As the present study focused on establishing large-scale modeling and integration framework for cultural heritage in space, it can be expected there will be many follow-up studies. The construction of large-scale cultural heritages often takes decades or even centuries. In addition to the spatial modeling and integration framework as explored in the present study, it is also of great significance for archaeological research and cultural education to study the modeling method and integration framework of large-scale cultural heritages in time. Another point our study makes is that the way of exploring scenes in large-scale cultural heritages should be different from that in small-scale heritages. How to allow the users to better perceive multi-scale spatial characteristics of scenes in large-scale cultural heritages may also be worth investigating.

\section{CONCLUSION}

Large-scale cultural heritage is a great concentration of national or regional history and culture with high research and conservation value. The current studies of the 3-D cultural heritage scene reconstruction mainly focused on local scale heritage due to a lack of modeling methods and scene organization for large-scale cultural heritage. We took the Grand Canal of China as an example for systematic reconstruction at a larger scale. We introduced a variety of remote sensing data sources for large-scale cultural heritage modeling, and then adopted a 3-D geographic global information system for large-scale 3-D scene organization and management. A four-step 3D virtual reconstruction was developed and successfully applied.

This study presented methods and key technology in aspects of data sources and model organization to solve reconstruction problem of large-scale cultural heritage with high efficiency and accuracy.

In data source aspects, the large regional remote sensing data and their multi-band images seem suitable for 3-D reconstruction of large-scale cultural heritage. In particular, existing various data sources were more than sufficient in providing the basal data for cultural heritage terrain modeling.

The 3-D spherical information system seems also suitable for organization and integration of large-scale 3-D scene.

The identical coordinate system was able to keep uniform all of the 3-D models in the virtual scene and provided integrated spatial analysis functionalities like area, round, distance calculation.

The tree level showed spatial relationship between the 3-D models. Parent-child nodes indicated include-spatial-relationship. The spatial extent of all child nodes did not exceed that of the parent's, or sibling nodes which are adjacent to each other, and thus it provided a basis for spatial relational query. 
The root node of the platform is the Earth model ensured cultural heritage virtual scene could be integrated without a spatial limit. This platform could import both the 3-D terrain model and the local heritage model of every large-scale cultural heritage to spherical grid and tile-hierarchy of the Earth model, and render them all together, which cannot be done in the other 3-D system.

Our study aimed at addressing the main challenges in virtual scene reconstruction of large-scale cultural heritage by application of remote sensing data and 3-D global GIS from spatial information field. This proved the efficiency and capability on the case study of the Grand Canal.

We believe this study can be valuable for regional and national cultural heritage protection, for Chinese government as an infrastructural research, and as a good reference for other large-scale cultural heritage around the world both in 3-D modeling and virtual scene organization.

\section{REFERENCES}

[1] Zahorik, P. and R.L. Jenison, Presence as being-in-the-world. Presence: Teleoperators and virtual environments, 1998. 7(1): p. 78-89.

[2] Gaitatzes, A., D. Christopoulos and M. Roussou. Reviving the past: cultural heritage meets virtual reality. in Proceedings of the 2001 conference on Virtual reality, archeology, and cultural heritage. 2001: ACM.

[3] Miyazaki, D., et al., The great buddha project: Modeling cultural heritage through observation, in Modeling from reality. 2002, Springer. p. 181-193.

[4] White, M., et al. ARCO-an architecture for digitization, management and presentation of virtual exhibitions. in Computer Graphics International, 2004. Proceedings. 2004: IEEE.

[5] Papagiannakis, G., A. Foni and N. Magnenat-Thalmann. Real-Time recreated ceremonies in VR restituted cultural heritage sites. in CIPA 19th International Symposium. 2003.

[6] Christou, C., et al. A versatile large-scale multimodal VR system for cultural heritage visualization. in Proceedings of the ACM symposium on Virtual reality software and technology. 2006: ACM.

[7] Cabral, M., et al. An experience using X3D for virtual cultural heritage. in Proceedings of the twelfth international conference on 3D web technology. 2007: ACM.

[8] Bruno, F., et al., From 3D reconstruction to virtual reality: A complete methodology for digital archaeological exhibition. Journal of Cultural Heritage, 2010. 11(1): p. 42-49.

[9] Núñez Andrés, A., et al., Generation of virtual models of cultural heritage. Journal of Cultural Heritage, 2012. 13(1): p. 103-106.

[10] Van Gorp, B. and H. Renes, A European cultural identity? Heritage and shared histories in the European Union. Tijdschrift voor economische en sociale geografie, 2007. 98(3): p. 407-415.

[11] Qiao-yi, C., The Grand Canal—On the Protection of Canal Culture. Journal of Hangzhou Teachers College, 2005. 3: p. 000.

[12] Clark, C.D., S.M. Garrod and M.P. Pearson, Landscape archaeology and remote sensing in southern Madagascar. International Journal of Remote Sensing, 1998. 19(8): p. 1461-1477.

[13] Katsianis, M., et al., A 3D digital workflow for archaeological intra-site research using GIS. Journal of Archaeological Science, 2008. 35(3): p. 655-667.

[14] Cao, C. and N.S. Lam, Understanding the scale and resolution effects in remote sensing and GIS. Scale in remote sensing and GIS, 1997. 57: p. 72.

[15] Chevrel, M., M. Courtois and G. Weill, The SPOT satellite remote sensing mission. Photogrammetric Engineering and Remote Sensing, 1981. 47: p. 1163-1171.

[16] Vrabel, J., Multispectral imagery band sharpening study. Photogrammetric Engineering and Remote Sensing, 1996. 62(9): p. 1075-1084.

[17] Schowengerdt, R.A., Remote sensing: models and methods for image processing. 2006: Academic press.

[18] Markham, B.L. and J.L. Barker, Spectral characterization of the Landsat Thematic Mapper sensors. International Journal of Remote Sensing, 1985. 6(5): p. 697-716. 
[19] Yang, C., J.H. Everitt and D. Murden, Evaluating high resolution SPOT 5 satellite imagery for crop identification. Computers and Electronics in Agriculture, 2011. 75(2): p. 347-354.

[20] DENG, J., et al., An Effective Way for Automatically Extracting Water Body Information from SPOT-5 Images. Journal of Shanghai Jiaotong University (Agricultural Science), 2005. 2: p. 198-201.

[21] Gonçalves, H., J.A. Gonçalves and L. Corte-Real, Measures for an objective evaluation of the geometric correction process quality. Geoscience and Remote Sensing Letters, IEEE, 2009. 6(2): p. 292-296.

[22] Richards, J.A., Remote sensing digital image analysis: an introduction. 2013: Springer.

[23] Guarnieri, A., F. Remondino and A. Vettore, Digital photogrammetry and TLS data fusion applied to Cultural Heritage 3D modeling. International Archives of Photogrammetry, Remote Sensing and Spatial Information Sciences, 2006. 36(5).

[24] Doneus, M. and Briese, C., Digital terrain modelling for archaeological nterpretation within forested areas using full-waveform laserscanning. In: M. Ioannides, D. Arnold, F. Niccolucci and K. Mania (Editors), The 7 International Symposium on Virtual Reality, Archaeology and Cultural Heritage VAST (2006)

[25] Gruen, A., Reality-based generation of virtual environments for digital earth. International Journal of Digital Earth, 2008. 1(1): p. 88-106.

[26] Gonzalez-Escribano, A., et al., An extensible system for multilevel automatic data partition and mapping. IEEE Transactions on Parallel and Distributed Systems, 2013. 99(1): p. 1.

[27] Akca, D., 3D Modeling of cultural heritage objects with a structured light system. Mediterranean Archaeology and Archaeometry, 2012. 12(1): p. 139-152.

[28] Carmel, D., N. Zwerdling and S. Yogev. Entity oriented search and exploration for cultural heritage collections: the EU cultura project. in Proceedings of the 21st international conference companion on World Wide Web. 2012: ACM.

[29] Alsadik, B., M. Gerke and G. Vosselman. Optimal Camera Network Design for 3D Modeling of Cultural Heritage. in the Proceedings of the XXII ISPRS Congress, Melbourne, Australia. 2012.

[30] Hug, C. and C. Gonzalez-Perez, Qualitative evaluation of cultural heritage information modeling techniques. Journal on Computing and Cultural Heritage (JOCCH), 2012. 5(2): p. 8.

[31] Papagiannakis, G., A. Foni and N. Magnenat-Thalmann. Real-Time recreated ceremonies in VR restituted cultural heritage sites. in CIPA 19th International Symposium. 2003.

[32] Pavlidis, G., et al., Methods for 3D digitization of cultural heritage. Journal of Cultural Heritage, 2007. 8(1): p. 93-98.

[33] Shimada, M., Ortho-rectification and slope correction of SAR data using DEM and its accuracy evaluation. Selected Topics in Applied Earth Observations and Remote Sensing, IEEE Journal of, 2010. 3(4): p. 657-671.

[34] Du, Q., et al. Digital Orthoimage Generation with Low Altitude Photogrammetric System Based on Unmanned Airship. in Image and Data Fusion (ISIDF), 2011 International Symposium on. 2011: IEEE.

[35] $\mathrm{Pu}, \mathrm{S}$. and G. Vosselman, Knowledge based reconstruction of building models from terrestrial laser scanning data. ISPRS Journal of Photogrammetry and Remote Sensing, 2009. 64(6): p. 575-584.

[36] Wells, S., et al. Rome Reborn in Google Earth. in Making history interactive, 37th proceedings of the CAA conference. 2009.

\section{AUTHORS}

\section{Dr. Jian Tan}

$\mathrm{Ph}$. D. in WEB Geographic information science

Affiliation: Key Laboratory of Digital Earth, Center for Earth Observation and Digital Earth, CAS (Visualization Technology department, International Centre on Space Technologies for Natural and Cultural Heritage under the auspices of UNESCO)

Research Interests: 3D Digitalization Aiding Cultural Heritage Archaeology Or Heritage Conservation; Digital Earth and Its Applications

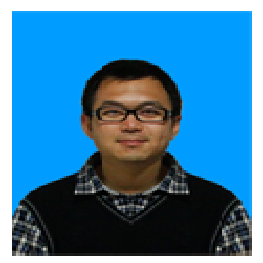

